\title{
Resolution of Hardy's Paradox within Spacetime Physics and the Ithaca Interpretation of Quantum Mechanics
}

\author{
Mohamed S. El Naschie \\ Dept. of Physics, Faculty of Science, University of Alexandria, Alexandria, Egypt \\ Email: Chaossf@aol.com
}

How to cite this paper: El Naschie, M.S. (2018) Resolution of Hardy's Paradox within Spacetime Physics and the Ithaca Interpretation of Quantum Mechanics. World Journal of Condensed Matter Physics, 8, 23-29. https://doi.org/10.4236/wjcmp.2018.82002

Received: February 24, 2018

Accepted: March 25, 2018

Published: March 28, 2018

Copyright $\odot 2018$ by author and Scientific Research Publishing Inc. This work is licensed under the Creative Commons Attribution International License (CC BY 4.0).

http://creativecommons.org/licenses/by/4.0/

\section{(c) (7) Open Access}

\begin{abstract}
By religiously adhering to physics in spacetime and taking the final verdict of N.D. Mermin's Ithaca interpretation of quantum mechanics seriously, Hardy's paradox is completely resolved. It is then concluded that logical and mathematically consistent physical theories must be put in spacetime related formalism such as noncommutative geometry and E-infinity theory to avoid quantum paradoxes. At a minimum, we should employ the philosophy behind consistent quantum interpretation such as that of the famous Ithaca interpretation of D. Mermin.
\end{abstract}

\section{Keywords}

Hardy's Paradox, Mermin's Ithaca Interpretation, Hardy's Quantum Entanglement, Noncommutative Geometry, E-Infinity Fractal-Cantorian Spacetime, Golden Mean Number System

\section{Introduction}

The present paper is devoted to resolving Hardy's famous paradox [1] [2] [3] [4] which gave us the precious jewel of quantum gravity, namely Hardy's probability of quantum entanglement of two quantum particles $P=\phi^{5}$ where $\phi=(\sqrt{5}-1) / 2$ [4] [5] [6] [7]. The paper was also written after undergoing a basic evolution in the mind of the author which made him extend his old belief that at a very deep level, physics and mathematics are two sides of the same coin to a new level convincing him that when devising thought experiments for fundamental mathematical theories, then philosophy and physics must be recalled to help mathematics from falling into the trap of paradoxes [8]-[13]. More often than not these traps are naturally encoded into our language which we use not only to 
communicate what we want to say to others, but is fundamental to our own thinking when we are talking to ourselves silently, i.e. thinking [8] [10] [14]. It is in this context that we have to recall Wittgenstein's critic of language and the connection between mental pictures, physics and language [15] which he wrote in the form of mostly aphorisms and published in his classic "Tractatus Logico-Philosophicus" [14].

In the present work, we will apply some of Wittgenstein's views to the superb paper "The Ithaca interpretation of quantum mechanics" by the eminent researcher and gifted educator, N. D. Mermin [4] and conclude that putting Hardy's paradox in E-infinity [9] [10] [11] [12] spacetime and Connes' noncommutative geometry [16], it ceases to be a paradox and harmonizes immediately with Mermin's conclusion and what we perceive as being reality [1] [2] [3] [4]. This is in essence what we will try to show in the following sections.

\section{Hardy's Paradox, the Golden Mean and Mermin's Resolution of the Problem}

Following Mermin's explanation and using his notation [4] we consider Lucien Hardy's beautiful gedanken experiment which incidentally was confirmed experimentally as far as quantum entanglement probability $\phi^{5}$ and found to be in excellent agreement with the theoretical results [11]. Hardy considered a two-two states system, for instance two photons with spin up and spin down. Let us call the two states A and B. Following the standard analytical procedure one arrives at the density matrix $|\Psi\rangle\langle\bar{\Psi}|$ with the normalized state projection [4]

$$
|\Psi\rangle=\frac{|2 R, 2 R\rangle-|1 R, 1 R\rangle\langle 1 R, 1 R \mid 2 R\rangle}{\sqrt{1-\langle 1 R \mid 2 R\rangle^{4}}}
$$

with three zero probabilities and a single non-zero probability given by [4]

$$
P(2 G, 2 G)=x^{2}\left(\frac{1-x}{1+x}\right)
$$

It turns out that the preceding Equation (1) holds the key not only to finding the famous fundamental probability of quantum entanglement $\phi^{5}$ but also to the resolution of Hardy's paradox [4] [11] [12] [13].

To show this we must point out first that the probability given by the vital Equation (1) attains a maximum value when $x$ is the golden mean $\phi=(\sqrt{5}-1) / 2$. At this point the reader could become confused because in the paper of Prof. D. Mermin, the golden mean is defined as $\phi=(\sqrt{5}+1) / 2$ and consequently $x$ must be replaced by $x=1 / \phi$. Indeed many authors do define the golden mean, also called golden section or divine proportion in this way. Many other authors, including the present one, reserve that name for $\phi=(\sqrt{5}-1) / 2=0.618033989$ and not $\phi=(\sqrt{5}+1) / 2=1.618083989$. This is of course a trivial point but could lead to confusion only due to habit and unfamiliarity with the golden mean number system but nothing more than that. Now 
Hardy's paradox arises from the reasoning given in the said Ref. [4] by concluding that the three zero probability which we found earlier on, leads to three conditional probabilities equal to unity. The point is then the obvious contradiction between the preceding result and all the related conclusions we can draw from our most important Equation (1) as explained lucidly in D. Mermin's paper [4].

In the same Ref. [4] and the references therein, the reader can find a detailed explanation of the conventional way of dismissing the paradox which we must admit to finding unsatisfactory and not consistent with our E-infinity theory nor with A. Connes' noncommutative geometry [16] [17]. We also note with a great deal of satisfaction that we are not alone in our perception of the conventional justification to dismiss the paradox and that Prof. D. Mermin reached the same conclusion based on his Ithaca interpretation of quantum mechanics [4]. To that end this interpretation does not harmonize with the classical dismissal of Hardy's paradox as we said earlier on. By contrast the Ithaca based resolution of the paradox consists of saying that the three conditional probabilities which are equal unity are meaningless and Mermin based himself in this respect on his anti-Baysian stance and his expressed opinion is that at some fundamental level unconditional joint objective probability has meaning while conditional probabilities have no meaning and concludes in this way that only correlation, that is to say only joint distribution are meaningful as objective reality [4].

In turn we declare here that we agree with the final conclusions of the Ithaca quantum interpretation based resolution but we think that there is a more direct and simpler way of reaching the same end via Equation (1) and our belief that there is an Achilles heel in Hardy's paradox and this weak point could be understood in terms of spacetime when merged with some basic philosophical ideas well known to positivistic "Weltanshaung" and Wittgenstein's critic of language laid down in his blue and brown books [15] as well as his Tractatus [14].

\section{From Wittgenstein to Zero Set, Empty Set, Spacetime, Wave Collapse and Hardy's Paradox Resolution}

As a theoretical physicist writing a paper for theoretical physicists, I do not want to indulge too much in the philosophy of Ludwig Wittgenstein and the Vienna circles of positivistic philosophy [14] [15]. However in the present paper at least it is important so I will be brief and give more or less an impressionistic picture and leave the reader to go over it once more with his brush and turn it into a realistic picture:

1) "The limits of my language mean the limits of my world [14] No. 5.6".

2) "The laws of physics, with all their logical apparatus (and mathematics) still speaks, however indirectly about the objects of the world [14] No. 6.3431".

3) "It is not how things are in the world that is mystical, but that it exists [14] No. 6.44".

4) "What we cannot speak about we must pass over in silence [14] No. 7".

The preceding four statements (aphorism) by Wittgenstein are taken from 
Ref. [14] where the numbers after the bracket are the those of Wittgenstein's small book [14].

In short we will let the four statements act on the usual statement outlining Hardy's paradox. In this circumstance, the author could not do better than quote Prof. D. Mermin verbatim on the crucial point which is stated on line number 14, page 563 of his classical paper [4]:

"Now consider the universe consisting of the pair of two-state systems ..."

The point is that we all think consciously or unconsciously of a universe having space at least and better still, to have spacetime for the two-state system plus an intelligent observer to observe the system in some sense not identical but somewhat similar to what Prof. J. A. Wheeler once proposed [18]. In other words we cannot simply reduce the entire universe to Hardy's set up and remain with the minimal reality about which many deep thinkers, including Wittgenstein, Wheeler and Mermin and a host of serious physicists and philosophers have thought deeply and then be astonished that even the simplest of mathematical deduction leads to paradoxes like those which plague orthodox quantum mechanics [1] [2] [4]. It is thus just as sinful and defect to ignore the objects of the real world as it is to ignore abstract mathematics. In fact the most fundamental mathematical theory which we have, namely transfinite set theory still speaks about collections of objects in a box and talks about quite mundane notions like full, zero and empty as fundamental theorems and axioms [11] [12].

We start from the empty set (sometimes misnamed null set which may be confused with the zero set via the German word null which means zero). This way we come to K. Menger, P. Urysohn, R. Penrose and A. Connes' ideas which give these mundane notions watertight, stringent mathematical notions to do calculations with [13] [16] [17]. That way we understand that the zero set is described by zero and $\phi=(\sqrt{5}-1) / 2$ while the empty set is given by minus one of $\phi^{2}$ [6] [10]. We can even go on and talk about the degree of emptiness of an empty set because a Cantor set [19] [20] could be made as thin as we want until it vanishes completely for minus infinity and $\phi^{\infty}$ which is not simply zero but insubstantial nothingness of the sort explained in the work of M. Heidiger and J. P. Sartre [21].

The preceding elucidation is neither abstract mathematics with no application to reality nor idle philosophy which keeps us entertained with no benefit to society as a whole. In fact it is the loathed wave collapse [5] [6] [20] [22] state vector reduction which in the trade goes under the name of measurement which can be understood best via the preceding set theoretical element. To do that all that we need is to identify the quantum particle with the zero set. It follows then that its "surface" is the empty set and that must be the quantum wave in the primordial form. Now measurement is a real act satisfying the criteria of reality of all physicists of the mold of Albert Einstein and the philosopher of the mold of Ludwig Wittgenstein [14] [15]. Consider an empty box and a sceptical realist wants to ascertain that the empty closed box is really empty. The only realistic way to do 
that is to penetrate the surface of the empty box (set) by any means. At the very moment we do that, we convert an empty box into a non-empty box due to penetrating this box. At a minimum the empty set will become a zero set if our examination (instrument) is infinitely subtle. The circle is now closed because finding the zero set is finding a quantum particle as opposed to the empty set quantum wave surface of the zero set [5] [11] [20]. Some readers may object to the preceding argument as being both unfamiliar, too simple and at the same time, maybe too abstract for the taste of many physicists who do not consider set theory to be part of physics. To that we can answer with what we said at the very beginning, namely that we are asking such deep and fundamental questions that are on a level where pure mathematics and pure physics are a unified entities [11] [20]. Never the less we think we can convince the sceptic by returning to Equation (1) which is our most powerful evidence for what we think is a fact, namely that quantum mechanics is just a spacetime theory as Newtonian mechanics and relativity. We wrote Equation (1) intentionally in the form of two brackets multiplication [10] [11] [12] [13]

$$
P=\left(\phi^{2}\right)\left(\frac{1-\phi}{1+\phi}\right)
$$

where we have substituted $\phi$ for $1 / 2$ of Mermin's notation. This was generalized several years ago using E-infinity theory to [19] [20] [22] [23]

$$
P=\left(\phi^{n}\right)\left(\frac{1-\phi}{1+\phi}\right)
$$

where $\mathrm{n}$ refers to the number of the two state sub-system, i.e. the number of the building blocks of our thought experiment set up. In addition $\phi$ represents the Hausdorff dimension of the zero set and consequently while the first bracket gives the probability of the quantum entanglement of $n$ quantum particles assumed to be theoretically divorced from any spacetime or surroundings, the second term is the intrinsic probability of spacetime itself where the inverse $(1+\phi) /(1-\phi)=4+\phi^{3}$ gives us the expectation value of the Hausdorff dimension of the corresponding fractal, self similar Einstein spacetime [19] [20] [22] [23]. From the above it becomes obvious that this space is characterized with the two dimensions, namely a topological dimension $D_{T}=4$ and a corresponding Hausdorff dimension $D_{H}=4+\phi^{3}$. Seen that way there can be no meaning for the second bracket having any value different from $4+\phi^{3}$. It is only the first bracket which can take infinitely many values depending on $n$ which in the case of Hardy's thought experiment is two $(n=2)$ quantum particles. Thus putting Hardy's set up into our realistic fractal Einstein spacetime would make all the three zero solutions and the conditional unit probability not only obsolete but they would virtually disappear into thin air. We also see that for a very large $\mathrm{n}$ which would tend to practically infinity, the first bracket would become almost zero so that the probability of entanglement for the entire universe with very large $N$ so to speak would be almost zero. This explains why in the classical 
world we could never measure any non-zero value for entanglement. From the above we see that all the three zeros of the lovely table shown on page 563 of Ref. [4] are not there and the only non-zero values are the golden mean to the power of $1,2,3,4$ and 5 while $\phi^{5}$ is the largest possible probability for entanglement except for the entanglement of one particle to spacetime which we interpret as the Unruh temperature and the case of $\phi^{3}$ which corresponds to Casimir effect measuring the difference between the zero set $\phi$ and the empty set $\phi^{2}$ given by $\phi-\phi^{2}=\phi^{3}$ as should be [11] [12] [13] [20] [22].

\section{Conclusions}

It seems that there are two types of fundamental theories: once which is clearly a spacetime dependent like that of Einstein gravity and another one where the involvement of spacetime is not explicitly obvious such as quantum mechanics. There is a price to pay when a theory is not clearly formulated in spacetime. This price is a large number of pseudo paradoxes popping up here and there in uncomfortable large numbers for our spacetime brain to live happily with. The work of Hardy contains a jewel coming out of a paradox. The jewel is his $\phi^{5}$ maximal quantum entanglement of two quantum particles while the paradox is not really a paradox because all become well when we think about Hardy's setup in spacetime. In fact, Hardy's $\phi^{5}$ leads to one of the most advanced spacetime theories which is Witten's fractal M-theory for which [24]

$$
\begin{aligned}
D & =1 / \phi^{5} \\
& =11+\frac{1}{11+\frac{1}{11 \ldots \ldots .}}
\end{aligned}
$$

This final result essentially says that a deep theory in physics is a theory in which physics meets mathematics (geometry), algebra, topology and forms something more real. Spacetime is a good place to start such a theory and may be the only consistent and paradox free place to start.

\section{References}

[1] Hardy, L. (1993) Nonlocality of Two Particles without Inequalities for Almost All Entangled States. Physics Review Letters, 71, 1665-1668. https://doi.org/10.1103/PhysRevLett.71.1665

[2] Aharonov, Y., Botero, A., Popescu, S., Reznik, B. and Tallaksen, J. (2002) Revisiting Hardy's Paradox: Counterfactual Statements, Real Measurements, Entanglement and Weak Values. Physics Letters A, 301, 130-138.

https://arxiv.org/abs/quant-ph/0104062 https://doi.org/10.1016/S0375-9601(02)00986-6

[3] Ho, M.-W. (2014) E-Infinity Spacetime, Quantum Paradoxes and Quantum Gravity. Journal of the Institute of Science in Society, 62, 40-43.

[4] Mermin, D. (1998) The Ithaca Interpretation of Quantum Mechanics. Pramana, 51, 549-565. https://doi.org/10.1007/BF02827447

[5] El Naschie, M.S. (2011) Quantum Entanglement as a Consequence of a Cantorian 
Micro Spacetime Geometry. Journal of Quantum Information Science, 1, 50-53. https://doi.org/10.4236/jqis.2011.12007

[6] Marek-Crnjac, L. (2011) The Common Sense of the Geometry of Quantum Entanglement. Fractal Spacetime and Noncommutative Geometry in Quantum and High Energy Physics, 1, 31-39.

[7] Ord, G., He, J.-H. and El Naschie, M.S. (Eds.) (2013) On the Shoulder of Giants. Fractal Spacetime and Noncommutative Geometry in Quantum and High Energy Physics, 3, 59-62.

[8] Aharonov, Y. and Rohrlich, D. (2005) Quantum Paradoxes: Quantum Theory for the Perplexed. Wiley-VCH Verlag, Weinheim.

https://doi.org/10.1002/9783527619115

[9] El Naschie, M.S. (2017) Looped Light on Dark Energy. Journal of Quantum Information Science, 7, 43-47. https://doi.org/10.4236/jqis.2017.72004

[10] El Naschie, M.S. and Olsen, S.A. (2011) When Zero Is Equal One: A Set Theoretical Resolution of Quantum Paradoxes. Fractal Spacetime \& Noncommutative Geometry in Quantum High Energy Physics, 1, 11-24.

[11] El Naschie, M.S. (2014) Hardy's Entanglement as the Ultimate Explanation for the Observed Cosmic Dark Energy and Accelerated Expansion. Journal of High Energy Physics, 1, 13-17. https://doi.org/10.11648/j.ijhep.20140102.11

[12] Marek-Crnjac, L. (2011) The Physics of Empty Sets and the Quantum. Nonlinear Science Letters B, 1, 8-9.

[13] El Naschie, M.S. (2017) Quantum Disentanglement as the Physics behind Dark Energy. Open Journal of Mircophysics, 7, 1-27. https://doi.org/10.4236/ojm.2017.71001

[14] Wittgenstein, L. (1961) Tractatus Logico-Philosphicus. Routledge and Kegan Paul, London.

[15] Wittgenstein, L. (1958) The Blue and Brown Books. Blackwell Publishing, Oxford.

[16] Connes, A. (1994) Noncommutative Geometry. Academic Press, San Diego.

[17] El Naschie, M.S. (1998) Penrose Universe and Cantorian Spacetime as a Model for Noncommutative Quantum Geometry. Chaos, Solitons \& Fractals, 9, 931-933. https://doi.org/10.1016/S0960-0779(98)00077-0

[18] Misner, C., Thorne, K. and Wheeler, J. (1973) Gravitation. Freeman, New York.

[19] El Naschie, M.S. (2004) A Review of E-Infinity and the Mass Spectrum of High Energy Particle Physics. Chaos, Solitons \& Fractals, 19, 209-236. https://doi.org/10.1016/S0960-0779(03)00278-9

[20] El Naschie, M.S. (2017) Elements of a New Set Theory Based Quantum Mechanics with Applications in High Energy Quantum Physics and Cosmology. International Journal of High Energy Physics, 4, 65-74.

[21] Sartre, J.P. (2012) Being and Nothingness. Open Road Media, New York.

[22] El Naschie, M.S. (2017) The Physics, Mathematics and Common Sense of Cosmic Dark Energy and Spacetime Extra Dimensions. International Journal of Innovations in Science and Mathematics, 5, 201-204.

[23] El Naschie, M.S. (2009) The Theory of Cantorian Spacetime and High Energy Particle Physics (An Informal Review). Chaos, Solitons \& Fractals, 41, 2635-2646. https://doi.org/10.1016/j.chaos.2008.09.059

[24] El Naschie, M.S. (2016) On a Fractal Version of Witten's M-Theory. Journal of Astronomy \& Astrophysics, 6, 135-144. 